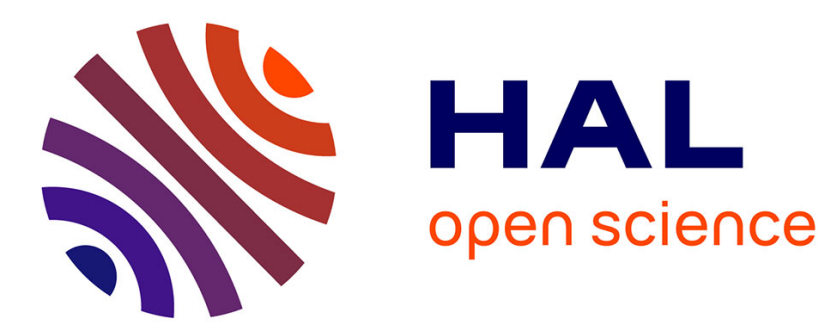

\title{
Héliopolis, création et assimilation d'une ville européenne en Egypte au XXe siècle
}

Mercedes Volait, J.-B. Minnaert

\section{To cite this version:}

Mercedes Volait, J.-B. Minnaert. Héliopolis, création et assimilation d'une ville européenne en Egypte au XXe siècle. Villes rattachées, villes reconfigurées: XVIe-XXe siècles, Presses de l'Université François-Rabelais, pp.335-365, 2003. halshs-00005272

\section{HAL Id: halshs-00005272 https://shs.hal.science/halshs-00005272}

Submitted on 25 Sep 2009

HAL is a multi-disciplinary open access archive for the deposit and dissemination of scientific research documents, whether they are published or not. The documents may come from teaching and research institutions in France or abroad, or from public or private research centers.
L'archive ouverte pluridisciplinaire HAL, est destinée au dépôt et à la diffusion de documents scientifiques de niveau recherche, publiés ou non, émanant des établissements d'enseignement et de recherche français ou étrangers, des laboratoires publics ou privés. 
Mercedes Volait, Jean-Baptiste Minnaert

(in Denise Turrel (dir.), Villes rattachées, villes reconfigurées: XVIe-XXe siècles, Collection "Villes et territoires”, Tours : Presses de l’Université François-Rabelais, 2003, p. 335-365)

\section{HÉLIOPOLIS, CRÉATION ET ASSIMILATION D’UNE VILLE EUROPÉENNE EN ÉGYPTE AU XXE SIÈCLE}

Héliopolis est aujourd'hui reconnue tant en Europe qu'en Égypte comme l'un des ensembles urbains contemporains parmi les plus cohérents et les mieux conservés de la métropole cairote. Cette création urbaine du XXe siècle est engagée depuis une dizaine d'années dans un processus de patrimonialisation qui, en 1997-1999, motiva une action d'expertise et la conception d'un projet de sauvegarde ${ }^{1}$, puis à partir de 2000 une recherche historique approfondie basée sur l'analyse du bâti et d'un fonds d'archives - celui de la Compagnie d'aménagement d'Héliopolis - d'une exceptionnelle richesse. Le substrat bâti et les archives se renseignant mutuellement, l'équipe composée autour du programme de recherche ${ }^{2}$ a eu ainsi la chance de pouvoir documenter dans le plus fin détail les rapports entre le quotidien de la fabrication de la ville et les doctrines qui trament le projet urbain. C'est dans cet écart entre doctrine et pratique, trop rarement analysé par les historiens de l'architecture et de la ville pour ce qui est des ensembles urbains sud-méditerranéens de la période contemporaine, que se situe la recherche ici présentée.

\section{Héliopolis au Caire}

C’est en 1905 que le baron Édouard Empain décide la création ex-nihilo d’une ville nouvelle qui accueillerait un habitat bourgeois construit aux normes techniques, fonctionnelles et sanitaires européennes, où l'on bénéficiera de soleil à profusion, d'air pur et d'un climat on ne peut plus $\sec ^{3}$. L'endroit choisi se situe à dix kilomètres au nord-est du Caire dans son extension de l'époque, en plein désert et, malgré ce que laisse entendre le nom même de cette nouvelle ville, aucun vestige archéologique n’est retrouvé à l'issue des fouilles commanditées par le baron. L'antique Héliopolis est en effet située beaucoup plus près du Nil. Le terrain est acheté à bas prix à l'État égyptien grâce aux entrées à la cour khédiviale de Boghos Nubar, fils du ministre Nubar Pacha, et associé d’Édouard Empain. Il était prévu initialement de créer plusieurs “ oasis” espacées les unes des autres, la plus éloignée du Caire accueillant la population la plus fortunée. Sur la concession obtenue, vaste de 25 kilomètres carrés, vierge de toute construction et de tout parcellaire, une première oasis est réalisée. Ce sera la seule.

La Cairo Electric Railways and Heliopolis Oases Company est fondée en 1906. Afin de

1 L'action a été menée dans le cadre d'un accord de coopération franco-égyptien, entre la General Organization for Physical Planning dépendant du ministère égyptien de l'Équipement, du logement et des nouvelles communautés urbaines et le laboratoire URBAMA de l'Université François-Rabelais de Tours, avec le soutien du Centre français de culture et de coopération de l'Ambassade de France au Caire, de la Direction de l'Architecture et du Patrimoine du ministère de la Culture et de la communication, de l'Association française d'action artistique, et de la MSH “ Villes et territoires" de Tours.

2 Placée sous la direction de Mercedes Volait, l'équipe est composée de Jean-Baptiste Minnaert, Claudine Piaton, Florence Troin, Xavier Rodier et Corinne Rupin. Un ouvrage monographique consacré à la fabrication et à l'évolution d'Héliopolis est actuellement en préparation (la publication en est prévue pour 2005).

3 En 1981, Robert Ilbert avait consacré une recherche à Héliopolis, principalement basée sur le dépouillement des procès-verbaux du conseil d'administration de la Compagnie d'aménagement. Robert ILBERT, Héliopolis - Le Caire 1905-1922. Genèse d'une ville, Marseille, Éditions du CNRS, 1981. 
contrôler étroitement la construction des infrastructures et la croissance de la ville, Édouard Empain met en place une efficace administration, basée à Héliopolis, ainsi qu’à Bruxelles où les permis de construire sont visés souvent de sa propre main. La Compagnie réalise les équipements publics (Heliopolis Palace Hotel, hippodrome, lieux de culte, marché), la voirie et les réseaux divers, et d'autres équipements liés à la fabrication de la ville, comme une pépinière pour ses plantations, une briqueterie, etc. La Compagnie réalise aussi des logements de toutes catégories ${ }^{4}$ : villas, immeubles de rapport, logements en bande, dont certains sont signés par des architectes français comme Alexandre Marcel, ou belges comme Ernest Jaspar, mais dont la plupart sont conçus par des architectes italiens, grecs, arméniens, syro-libanais, égyptiens. La construction des logements est concédée à des sociétés immobilières : la Société française d'entreprises en Égypte dirigée par Alexandre Marcel ; la Société des travaux publics du Caire, dirigée par Boghos Nubar.

L'éloignement du Caire est pensé non comme un inconvénient mais comme un avantage : l'homme d'affaires belge, qui a obtenu la concession du tramway du Caire en 1894, fait immédiatement aménager deux lignes : un "métro" (en fait une ligne de chemin de fer électrifiée) et deux lignes de tramway. Ces liens rapides à la métropole comptent parmi les atouts commerciaux de l'entreprise et fondent tant son identité que sa crédibilité. Héliopolis est assurément une ville-satellite par sa situation, son réseau de transports et le fait qu'elle compte rapidement un taux d'emplois locaux suffisant pour ne pouvoir être qualifiée de banlieue-dortoir du Caire. Héliopolis est imaginée aussi comme une station climatique de luxe, si l'on en croit du moins l'abondante publicité qui est faite lors de son lancement. Et, si elle ne peut passer pour une cité-jardin, nombreuses sont les ambiguïtés sémantiques qui tendent en partie à la désigner telle pour en retirer un bénéfice d'image ${ }^{5}$. Au demeurant, la ville comptera, en 1937 un peu plus de 30000 habitants, chiffre considéré comme optimal par Ebenezer Howard pour une cité-jardin, ce chiffre étant proche aussi de celui que Tony Garnier préconise dans son projet de cité industrielle (1901-1917).

\section{Héliopolis, ville de lotissement}

Si les concepteurs d'Héliopolis ne peuvent avoir méconnu la cité-jardin de Letchworth (1904), la banlieue-jardin de Hampstead (1907), ni les textes d'Howard et d'Unwin, l'urbanisation d'Héliopolis se situe plutôt dans la tradition, alors presque séculaire, du lotissement avec cahier des charges. Parmi d'incontournables exemples, probablement connus du baron Empain, on ne peut manquer de citer, rien que pour la France, Maisons-Laffitte ${ }^{6}$ lancé par le banquier Jacques Laffitte en 1833, ou Le Vésinet ${ }^{7}$ aménagé à partir de 1858. Il est par ailleurs avéré que les collaborateurs d'Empain ont médité l'exemple de Brooklyn, l'une des premières banlieues de commuters (créée à partir 1814), et il est probable qu'ils aient connu quelques villes-satellites américaines comme Gary, construite à quarante kilomètres de Chicago à partir de 1906.

L’intérêt historique d'Héliopolis tient en partie à ce que ses promoteurs ont eu la totale

\footnotetext{
4 En raison de la crise immobilière et financière de 1907, les acquéreurs se sont fait longtemps attendre. Aussi la Compagnie construisit-elle plus de sept cents immeubles et villas, qu'elle loua et, parfois, vendit. Ce n'est qu'à partir des années vingt que la construction privée relaya véritablement l'effort constructif de la Compagnie.
}

5 Les immeubles collectifs à coursives sont appelés par la Compagnie des “ Garden-cities ”.

6 Dominique Hervier, Sophie Cueille, Maisons-Laffitte. Parc, paysage et villégiature, 1630-1930, Inventaire général des monuments et des richesses artistiques de la France, Direction régionale des affaires culturelles d'Ilede-France, Paris, APPIF, 1999.

7 Dominique Hervier, Sophie Cueille, intr. François Loyer, Le Vésinet - Modèle français d'urbanisme paysager 1858-1930, Cahier de l'Inventaire $n^{\circ} 17$, Paris, Ministère de la culture et de la communication, Inventaire général des monuments et des richesses artistiques de la France, 1989. 
maîtrise du foncier et que, de surcroît, les seules règles d'urbanisme sont, initialement, celles qu'édicte et fait respecter la Compagnie, dépassant largement par leur détail les lois égyptiennes. La maîtrise du foncier et la liberté de composition étant totales, les intentions et la doctrine qui sous-tendent le projet urbain peuvent être d'autant plus explicites. Cependant, la mise en œuvre souffre d'approximations, dans le tracé du réseau viaire notamment, qui témoignent soit d'un manque de rigueur en phase opérationnelle (celle-ci semblant avoir parfois devancé le plan d'urbanisme, si tant est qu'il y en ait eu véritablement un), soit de précoces rapports de force entre le projet initial et les desiderata de propriétaires influents, arbitrés au bénéfice de ces derniers. La Compagnie, en effet, a toujours préféré les accords amiables, parfois à son détriment, plutôt que de nouer trop de contentieux et voir sa réputation en souffrir aux yeux des acquéreurs présents et futurs qui feront ou non le succès de la ville.

L'espace héliopolitain est conçu comme une scénographie urbaine ${ }^{8}$ qui se déroule le long des rues sinueuses et commerciales où passe le tramway. Celles-ci sont bordées d'élégants immeubles d'esthétique mauresque dont les arcades ménagent une ombre indispensable tout en déclinant l'incontournable modèle parisien de l'artère commerciale de prestige qu'est la rue de Rivoli. La scénographie est aussi axée par la large avenue des Pyramides, un parkway structuré en deux chaussées séparées par un terre-plein central verdoyant et arboré auquel font écho les jardins privés devançant les constructions à l'alignement ${ }^{9}$. De cette forme viaire inventée aux États-Unis, l'urbaniste et paysagiste Jean Claude Nicolas Forestier vante à la même époque, dans l'espace culturel français, la fonctionnalité et l'agrément ${ }^{10}$. La perspective de l'avenue vise vers le sud-ouest les lointaines pyramides de Giza ; elle s'achève au nord-est par la monumentale et très catholique basilique réalisée en 1913 par Alexandre Marcel, transposition à l'échelle un quart de Sainte-Sophie d'Istanbul et véritable centre géographique de la ville nouvelle, au-delà duquel l'avenue se scinde en deux rues divergentes. À cette sorte de cardo répond le decumanus que constituerait l'avenue Général Baron Empain dont les perspectives sont calées au nord-ouest par l'hippodrome et au sud-est par la villa du baron, improbable et fulgurant caprice néo-khmer ou néo-hindou construit par Alexandre Marcel en 1907-1911 en béton armé d'après les brevets de l'entrepreneur français François Hennebique. Comme dans la tradition urbanistique italienne et française forgée depuis le XVIe siècle et perpétuée sous Haussmann, les monuments jouent un rôle cardinal, focalisant, dans un jeu de pleins et de vides, la perspective des voies, et contre-pointant l'alignement des constructions privées qui leur servent d'écrin.

Les rues et les avenues sont hiérarchisées suivant la méthode du zonage, éprouvée depuis alors près de quarante ans aux États-Unis, puis en Allemagne, notamment. Le plan général de lotissement élaboré par la Compagnie codifie leur affectation (exclusivement résidentielle ou résidentielle et commerciale), leur calibre (largeur de la chaussée et des trottoirs), les matériaux de revêtement (type de bordures et de revêtement des trottoirs), l'essence des arbres qui sont plantés à l'alignement (ficus, jacarandas, palmiers), la hauteur des constructions qui les bordent, la position de ceux-ci à l'alignement ou devancés d'une zone de non-bâtisse

\footnotetext{
8 Pascal Garret, “Le passage à l'échelle urbaine : Héliopolis et ses mythes ” ; Anne VAn Loo, “ Ernest Jaspar à Héliopolis 1905-1916”, dans Mercedes Volait (dir.), Le Caire-Alexandrie, architectures européennes 18501950, Le Caire, CEDEJ / IFAO, 2001.
}

9 L’avenue des Pyramides a perdu depuis les années soixante son caractère de parkway : la ligne de tramway déviée en 1956 a remplacé le verdoyant terre-plein central et les jardins privés à l'alignement ont été remplacés par les excroissances de commerces qui se sont implantés sur cette voie initialement résidentielle.

10 Jean Claude Nicolas Forestier, Grandes villes et systèmes de parcs, (1908), reproduit dans : Jean Claude Nicolas Forestier, Grandes villes et systèmes de parcs France, Argentine, Maroc, présentation de Bénédicte Leclerc et Salvador TARragò i Cid, Paris, Norma, 1997. Henri Prost au Maroc à partir de 1913 utilisera largement cette forme d'espace. 
affectée à un jardin et dont la profondeur, ainsi que la hauteur de la clôture, sont également réglementées, suivant des dispositions très proches, l'idéologie mise à part, de celles que Charles Fourier évoquait dans ses utopies ${ }^{11}$. Ce zonage est aussi social. Le sud et l'est d'Héliopolis (en particulier le quartier de Korba au sud-est) accueillent dans d'opulents immeubles de rapport et d'élégantes villas une population bourgeoise et cosmopolite. À l'opposé, au nord-ouest, une "cité indigène" est construite pour loger la population autochtone, de condition très modeste, travaillant à la construction et à l'entretien de la ville ou au service domestique de la population bourgeoise. Les autres parties sont occupées par une population intermédiaire - en particulier des fonctionnaires du gouvernement égyptien, pour lesquels deux programmes de construction de logements sont lancés par la Compagnie en 1907, puis à partir de 1920 - logée dans des “villas ” qui peuvent atteindre trois niveaux et comporter autant d'appartements, dans des immeubles collectifs à vérandas et à coursives (dont l'impropre dénomination de "garden-cities" était sans doute volontaire), ou encore dans de simples mais coquettes maisons jumelées et “villas accolées ”, évoquant le row housing britannique.

Des cahiers des charges répartissent et modulent, à l'échelle de chaque parcelle, les prescriptions fonctionnelles, volumétriques et esthétiques du plan général de lotissement, et s'appliquent suivant la situation de la parcelle : dans une zone de villas ou dans une zone autorisée aux commerces. Les cahier des charges, d’une part, réglementent le rapport de la construction à l'espace public : ils précisent l'éventuelle zone de non bâtisse et, le cas échéant, fixent les dimensions de la clôture et la végétalisation de celle-ci ${ }^{12}$. Ils déterminent aussi la hauteur maximale de la construction, la surface bâtie maximale par rapport à celle de la parcelle, ainsi que, dans certaines zones, la couleur de la façade. Enfin, ils imposent des normes de salubrité et des dispositifs d'hygiène aussi draconiens que peut le faire la législation britannique qui passe alors pour la plus avancées en matière de salubrité de l'habitat $^{13}$. La formulation de ces cahiers des charges est d'autant plus rigoureuse et le respect

11 “Les clôtures et séparations ne pourront être que des soubassements, surmontés de grilles ou palissades qui devront à la vue au moins deux tiers de leur longueur, et n’occuper qu'un tiers en pilastre et palissades. [...] L'espace d'isolement doit être au moins égal à la demi-hauteur de la façade devant laquelle il est placé ; soit sur les côtés, soit sur les derrières de la maison. [...] L’isolement sur les côtés sera au moins égal au huitième de la largeur de la façade sur rue. [...] Les rues devront faire face à des points de vue champêtre, ou à des monuments d'architecture publique ou privée : le monotone échiquier en sera banni. Quelques-unes seront cintrées (serpentées), pour éviter l'uniformité. Les places devront occuper au moins 1/8 de la surface. Moitié des rues devront être plantées d'arbres variés dans chacune. Le minimum des rues est de neuf toises ; pour ménager les trottoirs, on peut, si elles ne sont que traverses à piétons, les réduire à trois toises, mais conserver toujours les six autres toises en clos gazonné ou planté et palissadé [...]. ” Charles Fourier, Traité de l'association domestiqueagricole, 2e édition, Paris, 1841. Cité par François Loyer, dans Dominique Hervier et Sophie Cueille, op. cit., p. 13.

12 Le cahier des charges $n^{\circ} 4$ (1912) stipule que la hauteur minimum des clôtures sera de 1,40 m. Sur les zones de non-bâtisse, les grilles surmonteront un soubassement en maçonnerie dont la hauteur par rapport au trottoir sera de 0,60 m maximum. La hauteur maximale de la clôture, soubassement et grille, sera de 2,30 m. Trois catégories de clôture sont indiquées sur le plan de lotissement : $5 \mathrm{~m}$ maximum (aspect à la volonté de l'acquéreur) ; 2,30 m maximum (aspect à la volonté de l'acquéreur sauf le long des voies publiques où les grilles sont obligatoires, comme dans les zones de non-bâtisse. La troisième catégorie est identique à la deuxième, sauf qu'elle peut être constituée de murs. Le plan des clôtures devra être soumis à la Compagnie. Le Caire, Archives de la Heliopolis Housing and Development Company.

13 Le cahier des charges $n^{\circ} 4$ (1912) stipule, notamment, que les cabinets d'aisance et salle de bains seront construits contre un mur extérieur, avec fenêtre, que la chasse d'eau aura une capacité de 10 litres maximum, que le sol sera couvert en matériaux lisses et imperméables, que les tuyaux de descente seront en fonte de 4 pouces de diamètre intérieur, que les descentes d'eau ménagères seront en fonte d'un diamètre intérieur de 3 pouces, que les descentes devront être placées hors les murs. Les tuyaux de descente de wc devront décharger dans une chambre d'inspection de 0,60x0,90 m reliée au drainage sans coupe-air, etc. Le Caire, Archives de la Heliopolis Housing and Development Company. 
de leur application d'autant plus sourcilleux que la Compagnie sait ensuite se montrer souple avec les contrevenants les plus influents.

\section{Types architecturaux}

L'esthétique architecturale que définit la Compagnie pour caractériser les espaces publics, les immeubles et villas qu'elle édifie elle-même, et ceux que construisent les particuliers sous son étroit contrôle réglementaire, est d'un pittoresque comparable à celui des villes balnéaires européennes, qu'elles soient de bord de mer ou, pour reprendre l'expression de François Loyer, de bord de ville. Bien entendu, pour cette soi-disant “ station d'hiver ” à l'usage d'une bourgeoisie éprise des standards domestiques européens, il paraît impératif de concevoir une architecture hygiénique, quitte à utiliser en ce site on ne peut plus aride des dispositifs architecturaux conçus en Europe contre l'humidité, et d'autres tout à fait inefficaces contre la canicule. Il ne s'avère pas moins nécessaire de produire une architecture techniquement et financièrement performante, par le très large usage qui est fait du béton armé (mis en œuvre suivant le système Hennebique) pour les planchers et les vérandas notamment, et, pour les murs porteurs, de peu coûteux moellons calcaires extraits des proches carrières de l'Abbassieh, ainsi que de briques silico-calcaires fabriquées presque à pied d'œuvre, dans une briqueterie créée par la Compagnie. C'est cependant le soin apporté à l'esthétique architecturale et urbaine, et sa variété, qui distingueront Héliopolis, non seulement par rapport aux concurrentes réalisations cairotes comme Hilwân ou Garden-city, mais aussi dans le cadre très concurrentiel du “marché” européen de la villégiature. D’où cette insistance de la Compagnie sur la qualité esthétique des projets, qu'elle exprime de manière si tatillonne lors de l'examen des demandes de permis de construire.

La Compagnie affirme fortement l'identité d’Héliopolis en premier lieu par la référence, non exclusive, à l'architecture fatimide et mamelouke de la vieille ville du Caire. Ce très élégant revival mauresque initié par Ernest Jaspar et Alexandre Marcel dans les premiers immeubles à arcades et à l'Héliopolis Palace Hotel (1907-1911), est décliné sur de nombreux édifices réalisés par la Compagnie et sur plusieurs palais. Il sert ainsi de modèle à un nombre non négligeable de constructions privées. Si l'on peut pister à Héliopolis une esthétique indosarasine qui rappelle à tout un chacun - et surtout aux Britanniques - que l'Égypte se trouve sur la route des Indes, c'est cependant le style Beaux-Arts français, triomphant en ce début de XXe siècle, qui constitue le bruit de fond de l'esthétique héliopolitaine. La sensibilité à l'Art nouveau n'est pas pour autant absente, et apparaît dans une consonance stile floreale en de nombreux détails de modénature moulés par des staffeurs souvent transalpins. À partir du milieu des années vingt, la référence à l’Art-déco puis, à partir des années trente, au mouvement moderne, donnent à de talentueux architectes comme Antoine Backh, Ézra Chammass, Charles Ayrout, Fahim Riad, Elias Tewfiq ou Max Balasiano, l'occasion de produire des œuvres qui n'ont parfois rien à envier à ce qui se fait alors de plus photogénique en Europe.

L’importance attribuée à la qualité des syntaxes architecturales, que celles-ci soient encouragées par la Compagnie comme le mauresque, ou unanimement goûtées comme le Beaux-Arts français puis l’Art-déco, démontrent la volonté du baron Empain et de ses collaborateurs de pratiquer un urbanisme total, dont témoigne par ailleurs le contrôle qu'ils exercent sur l'espace public, notamment dans l'esthétique des clôtures, et dont rendent compte aussi l'unité et la qualité du mobilier urbain. Il est par conséquent logique qu'au delà de l'esthétique de leurs façades, les constructions réalisées par la Compagnie se conforment à des types censés garantir la cohérence du paysage urbain. Cependant, leur grand nombre, ainsi que celui de leurs variantes (le tout dépasse les 200), révèlent à l'analyse des archives un singulier manque de logique. 
Ce n'est donc pas à travers les types architecturaux définis par la Compagnie que l'on peut reconstituer la typologie du bâti héliopolitain, mais plutôt dans les déclinaisons de modèles architecturaux qui s’inscrivent dans le zonage fonctionnel et social précédemment décrit. Ces modèles sont britanniques, belges et français et sont largement publiés à l'époque dans les revues tant européennes qu'égyptiennes. À ce stade de notre recherche, ils restent à pister et à préciser. Car les bourgeoises "villas" (terme qui désigne une construction de moins de trois étages et d'autant de logements), les immeubles de rapport à vérandas, les row-housings, les villas jumelées, notamment celles qui sont construites à partir de 1920 pour loger 600 fonctionnaires du gouvernement égyptien, parlent à l'historien de l'architecture européenne aussi clairement que s'il parcourait les banlieues de Londres, de Bruxelles ou de Paris ; seul l'exotisme de leur esthétique les éloignant de leurs référents nord-méditerranéens.

À travers ces types architecturaux, la Compagnie et les architectes qui construisent à Héliopolis se posent de manière plus ou moins adroite la question de l'adaptation du bâti aux usages locaux. Pour rester bref, nous n'évoquerons ici que le cas des salamliks, ces pièces de réception centrales et aveugles (ce qui garantit leur fraîcheur) adaptées aux habitudes de vie des Égyptiens et Levantins, fort nombreux à venir vivre à Héliopolis, et que les architectes ont insérés non sans habilité dans la trame constructive de logements pourtant tournés vers l'extérieur, à l'Européenne, par leurs vastes baies et larges et profondes loggias ou vérandas.

La diversité des formes et syntaxes architecturales inspirées de l'histoire de l'architecture européenne ou arabe relève de l'éclectisme et non de l'historicisme ${ }^{14}$. Les architectures mauresques d'Ernest Jaspar et d'Alexandre Marcel sont un heureux mélange des multiples aires ou périodes de l'architecture du monde musulman : fatimide, mamelouke, mais aussi andalouse, ottomane, voire moghole ou persane. Quant au style Beaux-Arts de nombreuses architectures héliopolitaines, s’il puise largement aux époques françaises de la Régence, de Louis XV et de Louis XVI, sa tenue doit beaucoup au talent des architectes de la Compagnie qui corrigent, voire reprennent, les projets présentés dans le dossier de permis de construire ; les projets initiaux parlant souvent une langue quelque peu impure. Quelle qu'en soit l'élégance, ces architectures, une fois réalisées, n'en arborent pas moins le plus souvent une esthétique composite. Par l'éclectisme de ses syntaxes, de ses techniques constructives, de ses normes fonctionnelles, de ses modèles architecturaux et urbains et, aussi, des idéologies qui sous-tendent sa création, Héliopolis constitue l'un des grands moments de cet éclectisme qui s’éteint progressivement au milieu du XXe siècle sous la poussée de l’idéologie moderne.

\section{Ancrages}

Par la diversité de ses typologies architecturales, par l'échelle de l'opération, par l'opiniâtreté mise à en contrôler l'esthétique, Héliopolis peut apparaître sans doute comme une réalisation tout à fait singulière. Il reste qu'à bien des égards, l'entreprise s'inscrit non moins étroitement dans le paysage égyptien ; de fait, elle en a d'emblée intégré plusieurs traits, au delà des emprunts aux répertoires décoratifs locaux. Si l'on resitue l'opération dans le contexte des pratiques urbanistiques qui avaient alors cours en Egypte, c'est en effet une parfaite continuité avec les modes de fabrication de la ville en vigueur depuis les années 1860 qui ressort. Pour la mise en œuvre des quartiers européens du Caire à partir de 1868, comme pour la construction de la cité balnéaire de Hilwân dix ans plus tard, ou par la suite de la banlieue-jardin de Ma'âdî (1905) ou encore du faubourg de Garden-City (1906), l'instrument principal de régulation des constructions fut le système de cahiers des charges plus ou moins détaillés, dont le respect conditionnait l'obtention d'un titre de propriété en bonne et due

14 Jean-Pierre Épron, Comprendre l'éclectisme, Paris, Norma, 1997. 
forme $^{15}$. On ne saurait donc parler à cet égard d'innovation de la part des dirigeants de l'Heliopolis Oases Company and Cairo Electric Railways (ci-après H.O.C. ou la Compagnie, comme elle est encore désignée en Egypte aujourd'hui).

Le zonage des populations et des fonctions qui organise l'espace héliopolitain ne lui est pas non plus spécifique. L’agencement des différents quartiers constituant Le Caire khédivial des années 1870 révèle tout autant une hiérarchie sociale et fonctionnelle assez stricte ${ }^{16}$. A Hilwân encore, le quartier “indigène” (destiné, comme à Héliopolis, au logement des domestiques et du petit personnel nécessaire au fonctionnement des services et équipements de la cité) fut, selon le même modèle, situé à l'écart des autres constructions (au sud-est, afin que les effluves, chassées par le vent du nord, ne les atteignent pas), et ses habitations obéissaient à des normes tout à fait différentes de celles applicables au reste des bâtiments ${ }^{17}$. Les situations d'“ entre soi ", produites par ces programmes de construction de logements socialement diversifiés, ne sauraient donc être analysées comme une invention de la compagnie belge. Et cette pratique a fait recette. Quels qu'aient été les changements politiques et sociaux traversés par l'Egypte au cours du XX $\mathrm{X}^{\mathrm{e}}$ siècle, le zonage social demeure à ce jour l'outil fondamental d'aménagement urbain et de planification spatiale, que l'on songe aux villes nouvelles des environs du Caire, découpées en secteurs pour bas revenus, moyens revenus ou revenus aisés, dotés d'habitations et d'espaces publics ad hoc ${ }^{18}$, ou aux dizaines de lotissements sécurisés haut de gamme qui ont poussé ces dix dernières années autour de la ville du 6-octobre ou en bordure des greens luxueux du plateau de Qatamiyya, dans les environs de la capitale égyptienne. A ces divers titres, on pourrait considérer qu’Héliopolis contenait en quelque sorte en germe les éléments de son assimilation.

\section{Un contexte de “ cohabitation"}

La création, puis le processus d'assimilation d'Héliopolis, prend place dans un contexte politique particulier, dont quelques traits nécessitent d'être brièvement rappelés. Jusqu'en 1914, l’Égypte constitue statutairement une province ottomane, assujettie donc à la Sublime Porte, à laquelle un tribut est annuellement versé, tout en étant occupée militairement par la Grande-Bretagne depuis 1882. De 1914 à 1922, l’Égypte se voit accorder le statut de Protectorat britannique, jusqu'à la déclaration unilatérale d’Indépendance proclamée par les Anglais en 1922 sous la pression d'un puissant mouvement national. A cette date, le pays devient de jure un Etat souverain et indépendant. Cette indépendance est certes relative du fait de différents points dits “ réservés”, liés en particulier au contrôle britannique du Soudan. En pratique, la mainmise britannique sur l'Égypte dut ainsi s'accommoder de différentes formes de " cohabitation" politique. Sur le plan législatif, il fallut en outre intégrer des aspects du droit coutumier - et les contraintes du système capitulaire ${ }^{19} .$. A l'inverse, l'Égypte

15 Pour une synthèse, cf. Mercedes Volait, “Making Cairo Modern : Multiple Models for a European-style Urbanism ”, dans Joe NAsR et Mercedes Volait, Urbanism - Imported or Exported ? Foreign plans and native aspirations, Chichester, John Wiley and sons, 2003.

16 Ibidem.

17 Elke Pflugradt, “La cité thermale d'Helwan en Egypte et son fondateur, Wilhelm Reil-bey ”, dans Catherine Bruant, Sylviane Leprun, \& Mercedes Volait, (dir.) Figures de l'orientalisme en architecture, $\mathrm{n}^{\circ}$ spécial de la Revue du Monde musulman et de la Méditerranée, n 73/74, 1996, p. 259-280.

18 Cf. François Ireton et Mercedes Volait (dir.), Les villes nouvelles en Egypte, Le Caire, CEDEJ, 1987.

19 Du nom des traités dits des Capitulations, en vertu desquels certains ressortissants étrangers, et les protégés des différentes puissances signataires, jouissaient d'une série de privilèges (exemptions d'impôt, juridictions spéciales...) au sein de l’Empire ottoman, cf. Joan Wucher KING, Historical Dictionary of Egypt, London, The Scarecrow Press, 1984, p 228-229. 
indépendante disposa d'une marge de manœuvre étroite.

Le nouveau statut obtenu en 1922 se traduisit concrètement par l'instauration d'un régime parlementaire dans le cadre d'une monarchie constitutionnelle et par la restauration de l'autonomie financière du gouvernement égyptien. Il ouvrit aussi la voie à l'égyptianisation à marches forcées de tous les secteurs de la vie politique, économique et sociale du pays ${ }^{20}$. Les premiers bénéficiaires en furent les élites cosmopolites, dont les membres des communautés “ levantines ”, avantagés par leur éducation dans les écoles chrétiennes et leur polyglottisme ${ }^{21}$, qui en faisaient des recrues de choix pour assurer la transition au niveau des postes intermédiaires dans l'administration comme dans le secteur privé - les fonctions de haut rang, quant à elles, avaient été continûment détenues par les élites égyptiennes ${ }^{22}$. Le nombre d'architectes, d'entrepreneurs et de propriétaires syro-libanais ou arméniens que l'on trouve associés à la construction d'Héliopolis trouve certainement là une causalité déterminante. Le principal d'entre eux, l'entrepreneur Habib Ayrout (1876-1956), d'une famille originaire d'Alep installée en Egypte depuis le XVII ${ }^{e}$ siècle, et ingénieur des Ponts et Chaussées de formation, fut actif dès l'origine de la création de la ville : ses premiers chantiers datent de 1906, et ses affaires, en association avec ses fils Charles (architecte) et Max (ingénieur) à partir de 1927, ne devaient pas cesser de prospérer ${ }^{23}$.

Une nouvelle page se tourne en 1952 avec le renversement du régime monarchique par les Officiers libres emmenés par Gamal Abd el-Nasser ; elle inaugure une ère marquée par des choix politiques et économiques radicalement différents de ceux qui avaient prévalu jusque là (instauration du système du parti unique, montée en puissance des élites militaires, programme d'industrialisation lourde, hypertrophie du secteur public, lourd endettement public...). Dans l'Egypte du premier $\mathrm{XX}^{\mathrm{e}}$ siècle, le changement de souveraineté fut une transformation inscrite dans la durée, et qui procéda par étapes.

\section{Assimilations}

La plus évidente assimilation d'Héliopolis est son assimilation géographique, qui l'a faite passer en quelques décennies d'une ville satellite quasi autonome située à $10 \mathrm{~km}$ du centre du Caire à l'un des quartiers de la capitale égyptienne. Il fallait traverser à l'origine des terres désertiques pour l'atteindre ; dès les années 1950, le trajet est entièrement urbanisé ${ }^{24}$. Comme le montre l'image satellitaire Spot de février $2000^{25}$, Héliopolis est désormais complètement noyé dans la tâche bâtie en continu de l'agglomération du Grand Caire. Le quartier en constitue l'un des trois grands pôles d'activités tertiaires, avec le quartier dit du centre-ville et celui plus récent de Muhandisîn, sur la rive gauche du Nil. C'est aussi le lieu quasi par excellence d'implantation du pouvoir puisque la Présidence de la République (située dans l'ancien Heliopolis Palace Hotel, restauré pour l'occasion au début des années 1990), l'étatmajor des armées et ses diverses branches, instituts et académies, la résidence du Président Hosni Moubarak lui-même, de ses ministres et de l'élite technocratique en règle générale, se

20 Afaf Lutfi AL-SAyyed Marsot, Egypt's Liberal Experiment: 1922-1936, Berkeley, University of California Press, 1977.

21 Sur les chawwam (originaires de la grande Syrie), cf. le témoignage de l’intérieur apporté par Edward W. SAID, A contre-voie : Mémoires, Paris, Le serpent à plumes, 2002. Sur la communauté maltaise, voir Anna et Pierre CACHIA, Landlocked islands : two alien lifes in Egypt, Le Caire, The American University in Cairo Press, 1999.

22 Cf. Magda Baraka, The Egyptian Upper Class between Revolutions, 1919-1952, Reading, Ithaca Press, 1998.

23 Jeanette Debbono-Ayrout, Une famille orientale : la chronique des Ayrout, Paris, 2001.

24 Jacques Besançon, “ Une banlieue du Caire : Héliopolis ”, Revue de Géographie de Lyon, vol XXXIII, 1958, p. $120-151$.

25 André Raymond (dir.), Le Caire, Paris, Citadelles et Mazenod, 2000, p. 18. 
trouvent à Héliopolis.

L'évolution de la toponymie est non moins parlante. La plupart des noms de rue originaux (simples numéros jusqu'en 1908) n'existent plus, à commencer par l'avenue Général Baron Empain, devenue al-Shahîd Tayyâr Nazîh Khalîfa (de l'aviateur martyr Nazîh Khalîfa) ou celle des Palais, désormais al-'Urûba (avenue de l'Arabité). L'hippodrome a été remplacé par un parc à l'anglaise, le “ Merry Land ”. La “ cité indigène ” se dénomme 'Izba al-Bustân", littéralement “la colonie des jardins”, et à l'exception de ses plus anciens habitants, peu d’héliopolitains savent qu'il s'agissait d'une sorte de coron composé de rangées de maisons en bande que de hauts murs isolaient du monde extérieur : d'après la tradition orale, on y accédait par un portail unique, fermé à partir du coucher du soleil et jusqu'à l'aube - son arche, désormais murée, est l'un des derniers vestiges des constructions initiales de la cité. Le quartier des villas est quant à lui connu sous le nom de Korba, en référence... à la " courbe ” très accusée que le trajet initial du tramway suivait à son arrivée dans cette partie de la ville mais cette étymologie est aujourd'hui à peu près oubliée de tous, d'autant que le tramway, depuis 1956, suit un autre parcours.

On peut évoquer ensuite l'assimilation sociale d'un quartier qui a progressivement tendu à déroger de moins au moins aux moyennes cairotes. En matière confessionnelle, par exemple, le peuplement initial d'Héliopolis fut marqué par une très forte présence chrétienne, composée pour l'essentiel de Syro-libanais grec-catholiques, d'Arméniens orthodoxes et latins, et d'Egyptiens coptes : “ Il convient de tenir compte du fait que la communauté copte est l'une des plus nombreuses d'Héliopolis " fut-il rappelé en 1920 aux responsables bruxellois de la Compagnie $^{27}$. L'équipement cultuel de la ville était à l'avenant : en 1940, on dénombrait 14 églises pour 4 mosquées et une synagogue (tardivement édifiée en 1928), destinées à une population de 40.000 habitants $^{28}$ - soit environ un rapport de une mosquée pour trois églises ; cette configuration distinguait très nettement Héliopolis des autres secteurs du Caire. Au recensement de 1986, la proportion de chrétiens dans les trois circonscriptions administratives qui recouvrent plus ou moins précisément le périmètre du quartier urbanisé par la H.O.C. (alBustân, Almâza et al-Muntaza), était de $18 \%$ pour $82 \%$ musulmans, alors que ces taux étaient respectivement de $10,5 \%$ et $89,5 \%$ pour le gouvernorat du Caire ${ }^{29}$. La présence chrétienne demeurait donc toujours plus nettement marquée à Héliopolis, mais cela se situait désormais dans un rapport moindre. Depuis, les mosquées et salles de prières paraissent s'être multipliées, dans les interstices entre parcelles, dans les zones de non-bâtisse, ou même dans les jardins publics, comme c'est le cas de la mosquée implantée dans le square Salah al-Din. La disparition de la rubrique relative à l'appartenance confessionnelle dans le recensement de 1996 (officiellement pour ne pas attiser la tension inter-confessionnelle) ne permet pas de prolonger la comparaison, mais tout porte à croire que le processus de “ normalisation ” par rapport aux moyennes cairotes s’est lentement mais sûrement poursuivi.

Contrairement à ce que l'on pourrait spontanément penser, de par l'origine belge du

26 La 'izba, traditionnellement translittérée en ezbah, désigne la colonie agricole établie par un gros propriétaire pour loger son personnel agricole (groupement d’habitations le plus souvent doté de tracés réguliers), et par extension son domaine, cf. J. LozAch et G. Hug, L'habitat rural en Egypte, Le Caire, IFAO, p. 1930, p. 156-157.

27 Lettre de la Direction générale d’HOC à Héliopolis au siège bruxellois de la Compagnie, 8 mai 1920 (Le Caire, Archives de la Heliopolis Housing and Development Company, Série des constructions particulières, dossier 90).

28 Décompte effectué à partir du plan d’Héliopolis au 1/2500ème publié par le service des Domaines de la H.O.C. en 1937 - document aimablement fourni par Robert Ilbert, que nous remercions vivement.

29 Statistiques établies par le Central Agency for Public Mobilization and Statistics, en charge des recensements décennaux, et aimablement fournies par l’Observatoire urbain du Caire contemporain à partir de la base EGIPTE. 
projet, Héliopolis ne fut nullement une enclave européenne au Caire (comme put l'être, par exemple, la banlieue jardin de $\mathrm{Ma}{ }^{\mathrm{a}} \hat{\mathrm{I}}^{30}$ ). Sa population fut d'emblée en grande majorité locale, et pas seulement en raison du nombre de domestiques ou d'employés des tramways résidant dans la “ cité indigène " et ses alentours. Les propriétaires égyptiens furent nombreux dès l'origine. Les acquisitions de terrain effectuées en 1910, par exemple, sont ainsi inscrites aux noms de Mahmoud bey Noushy, Ahmed Aly Abou Hendi, Habib Makhlouf, Elisabeth Guirgis, Allegra Menahem, Habib bey Zein, Hanna bey Sabbagh, Louis Adem, Moursi bey Ibrahim, tous sujets locaux de diverses confessions ${ }^{31}$. L'une des premières constructions particulières de la ville est un étonnant manoir néo-gothique, érigé en 1911 par l'avocat Me Abdel Kerim Raouf, rue Ménès, sur les plans de l'architecte égyptien Sayyid Metwalli ${ }^{32}$. On peut encore mentionner la dame Zeinab Hanem, fille d'Ibrahim Pacha Adham, d'une famille de hauts fonctionnaires d'origine turque ${ }^{33}$, qui commanda en 1921 aux architectes Guido Gavasi et Guiseppe Tavarelli, alors associés, un monumental palais néo-classique, flanqué du traditionnel salamlik des résidences ottomanes (pavillon de réception des hôtes étrangers à la famille) $)^{34}$. Le juriste Tewfik bey Henein fut le promoteur à partir de la même année de plusieurs villas, dont celle destinée à sa propre habitation ${ }^{35}$. Une estimation évalue à $50 \%$ d'Egyptiens, pour 30\% de Syro-libanais et 20\% d'Européens la composition “ nationale ” des habitants d'Héliopolis en $1925^{36}$. En 1937, Héliopolis représente le premier quartier de résidence de la haute-bourgeoisie musulmane du Caire : 16\% de ses membres y ont élu domicile, alors que les autres quartiers huppés en regroupent moins de $10 \%^{37}$. Les adresses héliopolitaines dans le bottin mondain égyptien de 1947 correspondent dans une écrasante majorité de cas à des personnes possédant des noms à consonance arabe ou égyptienne, le plus souvent avec des prénoms musulmans ; 63\% des 292 adresses répertoriées correspondent à des membres de la haute-bourgeoisie autochtone ${ }^{38}$. Dans les rangs de ces notables, on trouve des rentiers ou négociants, tel Yacoub bey Bibawi Attia (1894 - 1975), exportateur en coton, originaire de Samalout (Haute-Egypte), sénateur depuis 1936 et commanditaire d'une belle villa moderniste signée en 1939 par Awad Kamal Fahmy, architecte DPLG ${ }^{39}$, mais la liste contient surtout une proportion notable d'anciens ministres (Mohamed Ali Allouba, Abdelkawi Ahmed, Abdelrahman Omar, Aly El-Sayyed Ayoub, Amin Anis, Abdelhamid Badaoui...), de hauts fonctionnaires, dont l'homme de lettres Ahmed Loutfi El-Sayyed,

30 Samir RaAfat, Maadi 1904-1962, Society and history in a Cairo suburb, Le Caire, The Palm Press, 1994

31 Liste établie à partir des 500 premiers dossiers de permis de construire dépouillés, Le Caire, Archives de la Heliopolis Housing and Development Company, Série des constructions particulières, dossiers 25, 53, 86, 315, 321, 335, 337, 339, 874.

32 Ibidem, dossier 67. Sur Sayyid Metwalli, cf. A. Wright (dir.), Twentieth Century Impressions of Egypt, Londres, Lloyd's, 1909, p. 394.

33 F. R. Hunter, Egypt under the Khedives, 1805-1879, from Household Government to Modern Bureaucracy, Pittsburgh, University of Pittsburgh Press, p. 82.

34 Le Caire, Archives de la Heliopolis Housing and Development Company, Série des constructions particulières, dossier 104.

35 Ibidem, dossiers 101, 249, 376, 407.

36 Robert Ilbert, “Heliopolis, colonial entreprise and town planning success ?" dans The Expanding Metropolis : Coping with the Urban Growth of Cairo, Aga Khan Award for Islamic Architecture, Singapore, Concept Media, 1985, p. 36-42.

37 Magda Baraka, op. cit., p. 111-113.

38 Ibidem.

39 Le Caire, Archives de la Heliopolis Housing and Development Company, Série des constructions particulières, dossier 1208 et Egyptian Christian Biographical Dictionary, Alexandrie : Dar Mari Mina, 1995, p. 87. 
ancien recteur de l'université du Caire (logé dans l'une des villas à dôme de la Compagnie), de cadres militaires (en raison de la proximité des casernes de Manshiat al-Bakri) et de parlementaires ${ }^{40}$. Ces derniers représentent le plus souvent des circonscriptions de province, de Moyenne-Egypte en particulier ; on peut imaginer dans ce cas que leur logement à Héliopolis était utilisé comme résidence secondaire dans la capitale. Au total, cette population préfigure déjà largement la réalité sociale d'aujourd'hui. Il y a là à nouveau une belle continuité.

Sur le plan juridique, l'assimilation institutionnelle d'Héliopolis opéra de façon plus brutale: dans la tourmente de Suez et des décolonisations, la Compagnie se trouva transformée par le décret présidentiel n 285 de 1960 en organisme public, sous le nom tout d'abord de l'Organisation publique pour l'Oasis d'Héliopolis (Mû'assassa 'amma li-dâhia misr al-gadîda), devenue Heliopolis Company for Housing and Development par le décret $n$ • 2907 de 1964, dénomination qu'elle a conservé à ce jour. C’est aujourd'hui une société du secteur public (filiale de la Compagnie nationale de construction et de développement) placée sous la tutelle du ministère du Logement, de l'Equipement et des nouvelles communautés urbaines, tout en jouissant d'une forte autonomie sous la direction d'un conseil d'administration ${ }^{41}$. La concession des tramways qui reliait Héliopolis au centre du Caire, et était exploitée par la Compagnie, expira quant à elle comme prévu en 1977. Le changement de statut de la Compagnie accéléra la vente de son domaine bâti, qui était demeuré très important : il ne représentait pas moins de 768 constructions en 1937, soit environ 45\% des bâtiments existants à cette date ${ }^{42}$. Si dès l'origine, la Compagnie avait cherché à vendre ses immeubles et villas, les amateurs avaient été cependant rares et il avait donc fallu se résigner à la location. A partir des années 1950, les ventes se firent en revanche plus nombreuses, et décollèrent véritablement après la nationalisation de $1960^{43}$. Aujourd'hui, la Compagnie ne gère plus guère qu'une vingtaine d'édifices dans le noyau historique de la ville et ses activités paraissent largement recentrées sur l'aménagement de nouvelles extensions, toujours plus éloignées sur le plateau désertique oriental, avec le projet d'une " nouvelle Héliopolis" (Heliopolis al-gadîda ou New Heliopolis) sur 2500 ha en bordure de la route d'Ismâ'îliyya et à proximité de la ville nouvelle d'al-Chourouq. Lancé à grands renforts de publicité, le projet s'enorgueillit de bénéficier des savoir-faire acquis à Héliopolis, comme d'en reproduire certains modèles d'habitation ${ }^{44}$.

Sur le plan du contrôle édilitaire, la situation apparaît plus complexe à décrypter. Ainsi, par exemple, la gestion des permis de construire est désormais du ressort des services techniques du Gouvernorat du Caire, alors qu'elle avait été jusque dans les années 1950 dans les prérogatives exclusives de la société d'Empain, mais celle-ci conserve cependant un droit de regard sur ces permis en vertu des conditions stipulées dans les cahiers des charges grevant chaque parcelle de son domaine. Un propriétaire désireux de bâtir aujourd’hui à Héliopolis

40 E.J. Blattner, The Egyptian Who’s Who, Le Caire, 1947. Par commodité, la graphie, démodée, des noms de l'annuaire a été conservée. Le Caire, Archives de la Heliopolis Housing and Development Company, Série des constructions particulières, dossier 4571.

41 Le Caire, Archives de la Heliopolis Housing and Development Company, Série des constructions particulières, dossier 9463, nouveau Cahier des charges $n^{\circ} 1$ régissant la vente de terrains à Héliopolis, art. 7 , 1965 et Heliopolis Company for Housing and Development, Past, Present... and Future and Comprehensive Urban Development, Le Caire, 1996.

42 Décompte effectué à partir du plan d’Héliopolis au 1/2500ème publié par le service des Domaines de la H.O.C. en 1937.

43 C’est ce qui apparaît sur la base des dépouillements effectués jusqu’à présent.

44 Entretien avec Salah Chehata, chef du bureau technique de la Heliopolis Housing and Development Company, juillet 2002. 
doit donc se plier à la fois à la réglementation générale de la construction et aux règles imposées par le cahier des charges correspondant à son lot. Cette situation avait été en quelque sorte anticipée par la Compagnie, par sa propre volonté ou celle des autorités : les cahiers des charges précisaient ainsi que "les constructions des maisons d'habitations et autres édifices restaient soumises aux règlements et prescriptions en vigueur ou à édicter, comme d'ailleurs et d'une façon générale, toutes les lois et règlements du Gouvernement édictés ou à édicter, sont applicables dans toute l'étendue de la superficie vendue "45. C'est ainsi que le règlement sur les constructions d'Alexandrie de 1923, puis le nouveau règlement d'édilité négocié pour les extensions d'Héliopolis en $1931^{46}$, furent opposables aux particuliers désirant bâtir dans le domaine de la Compagnie. Puis ce fut le tour de la première loi générale encadrant l'urbanisme adoptée en Egypte en 1940 (loi nº 52 de 1940 relative à l'établissement des plans de lotissement), puis de la loi $n^{\circ} 93$ de 1948 sur les travaux de construction. En 1959, la direction générale de la voirie (direction dite du Tanzim) de la Municipalité du Caire rappelait, par la voix de son inspection d'Héliopolis, que la Compagnie s'était engagée à faire respecter les règlements généraux édictés ou à édicter, et devait donc appliquer, par exemple, l'art. 16 de la loi $n^{\circ} 656$ de 1954 interdisant la construction de passages et d'impasses dans la ville du Caire, et donc l'implantation de bâtiments additionnels en fond de parcelle ${ }^{47}$. Héliopolis est désormais soumise au nouveau code de l'urbanisme $\left(n^{\circ} 3\right.$ de 1982) et à la réglementation générale des constructions, sauf disposition contraire : en 1997, un périmètre du noyau historique d’Héliopolis, incluant la grande avenue al-‘Urûba, fut soustrait par décret ( $\mathrm{n}^{\circ} 500$ de 1997) aux dispositions de la réglementation générale sur les travaux de construction (loi $n^{\circ} 101$ de 1996 amendant la loi $n^{\circ} 106$ de 1976) afin de ne pas altérer sa physionomie. Sur les parcelles riveraines de l'avenue al-'Urûba, la hauteur maximale des constructions fut ainsi limitée à $8 \mathrm{~m}$ de hauteur (deux étages) en front de rue sur une profondeur de $80 \mathrm{~m}$ (retrait de $10 \mathrm{~m}$ inclus) ; les hauteurs demeurèrent plafonnées dans le reste de la zone dérogatoire à une fois et demi la largeur de la rue, avec un maximum de $35 \mathrm{~m}^{48}$. En 1993, un arrêté du gouverneur du Caire avait limité les hauteurs à Héliopolis à des immeubles composés d'un sous-sol, d'un rez-de-chaussée et de 6 étages ${ }^{49}$. La mesure intervenait deux ans après qu'un autre arrêté du même Gouvernorat ait interdit la construction d’immeubles en remplacement des villas ${ }^{50}$.

\section{Subversions et continuités}

Cet empilement de règles et de directives, éventuellement contradictoires et à force exécutoire variable, entretient une certaine confusion, propice à toutes sortes de subversions, en pleine légalité, des réglementations de la construction. Un bel exemple en est fourni par la pratique de surélévation des édifices, qu’il s’agisse d’immeubles ou de villas. Dès l'origine de la ville,

45 Le Caire, Archives de la Heliopolis Housing and Development Company, Série des constructions particulières Article 5 du cahier des charges nº 4 modifié, 1923, Dossier 51.

46 Mahboub Sabry Maнвоub, “ Cairo, some notes on its history, characteristics and town plan ”, dans Journal of the Town Planning Institute, vol. 21 (1934/35), p. 288-302 ; Mercedes Volait, "Town Planning Schemes for Cairo conceived by Egyptian Planners in the "Liberal Experiment" Period", dans Middle Eastern Cities 19001950: Public Spaces and Public Spheres in Transformation, J. Skovgand-Petersen (ed.), Proceedings of the Danish Intitute in Damascus, I - 2001, p. 44-71.

47 Le Caire, Archives de la Heliopolis Housing and Development Company, Série des constructions particulières, dossier 52.

48 Il s’agit du décret n500 de 1997, en date du 2 mars 1997, modifiant la loi n² 2104 de 1996 portant dispositions sur les hauteurs dans différents quartiers du Caire, Ibidem, dossier 6732.

49 Arrêté du gouverneur du Caire nº 349 en date du 4 mai 1993, Ibidem, dossier 6761.

50 Arrêté du gouverneur du Caire, n³9 du 9 mars 1991, Ibidem, dossier 321. 
bien des bâtiments avaient été mis en oeuvre par étapes, car peu de propriétaires avaient les moyens de mener à bien l'intégralité de leur projet de construction dans un délai limité ; le plus souvent prévues à l'avance, parfois imaginées après coup, ces surélévations reprenaient en règle générale l'esthétique des façades existantes et s’intégraient sans trop de mal, au point d'être à peu près indécelables à un œil non averti. Mais c'est un autre type de surélévations que devait connaître Héliopolis à partir des années 1970. Arguant de la crise du logement dans la capitale égyptienne et de la différence avec les normes, plus généreuses, permises par la législation générale de la construction, le Conseil d'administration de la Compagnie avait adopté en 1972 une résolution ( $\mathrm{n}^{\circ} 8$ en date du 20 février 1972) autorisant une augmentation du plafond des hauteurs sur tout son domaine, moyennant contrepartie financière des propriétaires souhaitant bénéficier de cette disposition. Le plafond applicable pouvait ainsi se trouver porté de 15 à 20m, contre paiement d'une majoration de $20 \%$ du prix du terrain. Quelques années plus tard (résolution en date du 26 août 1975), la possibilité d'un nouveau dépassement jusqu'à 28 m était offerte aux propriétaires, la majoration étant cette fois fixée à $12,5 \%$ du prix du terrain pour chaque étage supplémentaire ${ }^{51}$. Par la suite fut également instituée une majoration pour l'installation de compteurs d'électricité additionnels.

Les effets concrets de ces mesures ne se firent pas attendre : les édifices non surélevés sont désormais l'exception à Héliopolis. Cette stratégie d'optimisation du capital foncier de la Compagnie représente à nouveau une ligne de continuité dans l'histoire du développement d'Héliopolis ; elle repose en outre sur un dispositif qui existait déjà dans les années 1940. L'un des derniers cahiers des charges rédigés avant la nationalisation (cahier 8A de 1947) prévoyait en effet la possibilité de majorations ou de minorations du coût d'acquisition d'une parcelle, selon l'utilisation qu'un propriétaire faisait de ses droits à bâtir. Lors de ventes dans le quartier des villas, par exemple, une apostille précisait en effet que le prix du terrain avait été fixé en considération de l'engagement formel pris par l'acquéreur de n'élever sur sa parcelle qu'une seule villa ne comportant qu'un appartement, mais qu'il lui était loisible de construire le second étage moyennant une majoration du prix du terrain, si la soumission des plans du second appartement à construire intervenait dans un délai de dix ans. Passé ce délai, la majoration n'était plus éligible ${ }^{52}$. C'est donc la lettre, sinon l'esprit, d'une disposition ancienne qui fut reprise pour la gestion des surélévations à Héliopolis. En revanche, si préserver jalousement l'esthétique de la ville avait pu être perçu, avant les années 1960, comme le meilleur garant du succès financier de l'entreprise, ce n'était désormais plus le cas. Aux surélévations si discrètes des origines succédèrent des additions d'étages à peu près dénuées de tout souci d’harmonisation avec le restant de l'édifice - et pour le coup, parfaitement identifiables au premier coup d'œil.

\section{Préservations}

Un dernier registre de l'intégration d'Héliopolis tient à son assimilation patrimoniale. Depuis les débuts de la décennie 1990, un souci conservatoire se manifeste de façon générale pour toute architecture appartenant à ce qu'il est convenu d'appeler l'Egypte moderne, c'està-dire en gros les années 1850-1950, période également connue comme étant celle de la “Belle Epoque”; et Héliopolis n’échappe pas à la règle. Les manifestations de cette redécouverte sont nombreuses. Des campagnes de presse ont été développées pour sauver de la destruction des palais et villas du Caire ou d'Alexandrie, des sites web ont été développés (à commencer par www.egy.com), des associations se sont créées ou sont en voie de l'être, telle l'association Turâth (Patrimoine) créée à l'initiative de l'ambassadeur Raouf al-Ridi afin de s’occuper spécifiquement du patrimoine récent. Le Conseil suprême des Antiquités a

51 Ibidem, dossier 1208.

52 Ibidem, dossier 1739. 
entrepris d'en classer différentes manifestations, en s'appuyant sur les dispositions de la nouvelle loi sur les Antiquités adoptée en 1983 (loi nº 117 de 1983). Cette loi considère en effet comme éligible au classement tout bâtiment vieux de plus de 100 ans (art. 1), tout en prévoyant de pouvoir protéger des édifices postérieurs, lorsque " l'intérêt national " le justifie $\left(\right.$ art. 2) ${ }^{53}$. Dans les faits, la liste des bâtiments datant des XIX et $\mathrm{XX}^{\mathrm{e}}$ siècles, classés au titre de l'une ou l'autre de ces dispositions, ne cesse de s'allonger: sur les 119 arrêtés de protection promulgués entre 1982 et 1997 pour des monuments de toutes époques, 53 concernent des édifices des deux siècles écoulés ${ }^{54}$. Deux bâtiments d'Héliopolis sont ainsi protégés depuis plusieurs années : la Villa hindoue du baron Empain (par arrêté du ministre de la Culture en date du 30 juin 1987, renforcé par décret du Premier ministre n 1297 du 6 août 1993) et l'Héliopolis Palace Hotel - rebaptisé en Palais de l’Arabité (Qasr al-'Urûba) -, par le décret du Premier ministre n 112 du 17 janvier 1997.

A partir de 1997, l'engagement personnel de Mme Moubarak, épouse du président égyptien, en faveur du patrimoine architectural et urbain de l'Egypte moderne s'est traduit par une multiplication des initiatives au sein des ministères et des collectivités territoriales. Par arrêté du 5 avril 1998, le gouverneur du Caire a créé un Comité consultatif pour la préservation des bâtiments des $\mathrm{XIX}^{\mathrm{e}}$ et $\mathrm{XX}^{\mathrm{e}}$ siècles, afin d'établir un inventaire des constructions méritant protection et de proposer les mesures susceptibles de favoriser leur conservation. Une première évaluation a montré qu'il ne subsistait plus au Caire que quelque 1900 villas au total, compte tenu de la pression foncière. Des inventaires par quartiers ont été ensuite entrepris : à Héliopolis, 350 édifices d’intérêt historique ou artistique ont été identifiés par une équipe de l’Université d'Helwân ${ }^{55}$.

En 1997, des études ont été également engagées dans une perspective d'aménagement urbain. La volonté de préserver les ensembles urbains où se situe l'essentiel du patrimoine récent de la capitale égyptienne a ainsi donné lieu à la réalisation d'études conduites par des équipes universitaires à la demande des autorités en charge de la planification urbaine, le GOPP (General Organization for Physical Planning). Dix quartiers du Caire aménagés au tournant du siècle et désormais considérés comme ayant un caractère historique ont été ainsi étudiés : 'Ayn Chams, Héliopolis, Garden City, centre-ville, Hilmiyya al-Gadîda, Rudâ, Zamalek, Giza et Ma’âdî́n .

Dès 1991, les pouvoirs publics ont en outre cherché à limiter le plus strictement possible la démolition des villas et leur remplacement par des immeubles de hauteur. Les mesures locales prises dans différents gouvernorats ont finalement conduit à l'interdiction pure et simple des démolitions de villas sur tout le territoire égyptien, à l’initiative de l'ancien

53 Egyptian Antiquities Organization, Law n¹17 of 1983 concerning the issuance of Antiquities’ protection law (with a preface by Dr. Ahmed Kadry), Le Caire, 1985.

54 Organisme généRAl des Antiquités, Bayân bil-âthâr al-musaggala (Etat des monuments classés), Le Caire, s.d. (c. 1997), 11p., document manuscrit. Pour les principaux exemples, cf. Mercedes Volait, “Introduction ”, dans Id. (dir.), Le Caire - Alexandrie, architectures européennes, 1850-1950, Le Caire, co-édition IFAO/CEDEJ, 2001, p. IX-XV, ainsi que Id., "Colonisation, mondialisation et patrimonialisation de l'espace bâti dans le Monde Arabe", in Questions sur le patrimoine architectural et urbain au Liban, Z. Akl et M. Davie (dir.), Académie libanaise des Beaux-Arts/URBAMA, Beyrouth/Tours, 1999, p. 29-50

55 Cf. Cristina Scarpocchi, Logiques, formes et mécanismes de la réhabilitation et du renouvellement du bâti à Héliopolis, document manuscrit, 2002 (enquête menée dans le cadre du programme de coopération francoégyptienne “Préservation du patrimoine contemporain dans le Grand Caire : le cas d’Héliopolis” mentionné supra, et avec le soutien du CEDEJ).

56 La partie documentaire de l'étude consacrée au centre-ville du Caire a fait l'objet d'une publication, cf. Soheir Zaki Hawas, Khedivian Cairo : Identification and Documentation of Urban Architecture in Downtown Cairo, Le Caire, Architectural Designs Center, 2001. Comme indiqué supra, celle sur Héliopolis est menée directement par le GOPP en coopération avec URBAMA. 
premier ministre, Kamal al-Ganzuri (décret $n^{\circ} 463$ de 1998). Une stricte limitation des hauteurs des constructions neuves dans les quartiers à caractère historique a également fait l'objet d'une série de lois, tel le décret n 500 de 1997 mentionné supra. Cet ensemble de mesures a été renforcé par l'ordonnance militaire nº 2 de 1998, en date du 21 juin 1998.

Ces mesures n’ont certes pas arrêté tout projet de démolition dans les principales villes égyptiennes. Il n'est que de se promener dans les rues du centre d'Héliopolis pour constater que les destructions se poursuivent, et que bien d'autres sont en projet, si l'on en juge le nombre de propriétés vidées de leurs occupants, et parfois déjà dépouillées de toutes leurs huisseries, dans l'attente d'une autorisation de démolir en bonne et due forme. En cas de rejet de la demande de permis de démolition, nombre de propriétaires recourent en effet aux tribunaux en plaidant l'insalubrité de leur bien pour cause de vétusté (artificiellement provoquée, le cas échéant) ou encore sa qualité d’immeuble et non de villa - ils obtiennent généralement satisfaction, mais la procédure requiert plusieurs années pour aboutir.

Fortement soumises à la pression des propriétaires privés, les autorités locales tentent pourtant de résister. Le gouverneur du Caire, Abdel Rehim Chehata, bloque ainsi depuis plusieurs années un projet d'aménagement de l'îlot des anciennes tribunes de l'hippodrome, qui proposait la destruction de toutes ses anciennes constructions et leur remplacement par des immeubles de grande hauteur à usage commercial et résidentiel. Et l'unique haute tour de plus de 30 étages qui défigurait violemment le paysage d'Héliopolis est, depuis l'été 2002, en cours de destruction - il est vrai pour des motifs plus sécuritaires (ses baies donnaient sur les locaux de la Présidence) que patrimoniaux.

De façon plus générale, la nostalgie du temps jadis touche Héliopolis, comme elle touche les anciens "quartiers européens" du Caire ; des œuvres de fiction, de parution récente, en témoignent amplement ${ }^{57}$. Cette nostalgie du temps jadis, qui est aussi critique du temps présent, recompose à sa façon l'histoire contemporaine du pays, désormais recentrée sur la monarchie égyptienne, ses fastes supposés et ses splendeurs "Belle Epoque " 58 , tandis que l'épisode colonial tend à être, sinon gommé, du moins confiné à un décor d'arrière-plan ou à quelques personnalités. Pleinement égyptienne aujourd'hui, même si ses habitants revendiquent une identité particulière, Héliopolis se souvient, sans déplaisir, du fameux “Baron”.

57 May al-Telmessany, Heliopolis, Le Caire, Dâr Charqiyyât, 2000 ; Alaa AL-Aswany, 'Imara Yacoubian [Immeuble Yacoubian], Le Caire, Madbuli, 2002.

58 La revue Misr al-Mahrussa, publiée par le publicitaire Maged Farag, offre une bonne illustration du phénomène actuel d’engouement monarchiste. 